\title{
Screening key genes and signaling pathways in colorectal cancer by integrated bioinformatics analysis
}

\author{
CHANG YU $^{1 *}$, FUQIANG CHEN $^{1 *}$, JIANJUN JIANG $^{2}$, HONG ZHANG $^{3,4}$ and MEIJUAN ZHOU ${ }^{1}$ \\ ${ }^{1}$ Department of Radiation Medicine, Guangdong Provincial Key Laboratory of Tropical Disease Research, \\ School of Public Health; ${ }^{2}$ Department of Thoracic Surgery, Nanfang Hospital, Southern Medical University, \\ Guangzhou, Guangdong 510515; ${ }^{3}$ The First Affiliated Hospital; ${ }^{4}$ School of Management, \\ University of Science and Technology of China, Hefei, Anhui 230026, P.R. China
}

Received September 20, 2018; Accepted April 24, 2019

DOI: $10.3892 / \mathrm{mmr} .2019 .10336$

\begin{abstract}
The aim of the present study was to identify potential key genes associated with the progression and prognosis of colorectal cancer (CRC). Differentially expressed genes (DEGs) between CRC and normal samples were screened by integrated analysis of gene expression profile datasets, including the Gene Expression Omnibus (GEO) and The Cancer Genome Atlas. Gene ontology (GO) and Kyoto Encyclopedia of Genes and Genomes (KEGG) pathway analysis was conducted to identify the biological role of DEGs. In addition, a protein-protein interaction network and survival analysis were used to identify the key genes. The profiles of GSE9348, GSE22598 and GSE113513 were downloaded from the GEO database. A total of 405 common DEGs were identified, including 236 down- and 169 upregulated. GO analysis revealed that the downregulated DEGs were mainly enriched in 'detoxification of copper ion' [biological process, (BP)], 'oxidoreductase activity, acting on $\mathrm{CH}-\mathrm{OH}$ group of donors, NAD or NADP as acceptor' [molecular function, (MF)] and 'brush border' [cellular component, (CC)]. Upregulated DEGs were mainly involved in 'nuclear division' (BP), 'snoRNA binding' (MF) and 'nucleolar part' (CC). KEGG pathway analysis revealed that DEGs were mainly involved in 'mineral
\end{abstract}

Correspondence to: Professor Hong Zhang, School of Management, University of Science and Technology of China, 96 Jinzhai Road, Hefei, Anhui 230026, P.R. China

E-mail: zhangh@ustc.edu.cn

Professor Meijuan Zhou, Department of Radiation Medicine, Guangdong Provincial Key Laboratory of Tropical Disease Research, School of Public Health, Southern Medical University, 1023 Shatai South Road, Guangzhou, Guangdong 510515, P.R. China

E-mail:1kzmj@smu.edu.cn

"Contributed equally

Key words: colorectal cancer, bioinformatics analysis, differentially expressed genes, biomarkers, prognostic absorption', 'nitrogen metabolism', 'cell cycle' and 'caffeine metabolism'. A PPI network was constructed with 268 nodes and 1,027 edges. The top one module was selected, and it was revealed that module-related genes were mainly enriched in the GO terms 'sister chromatid segregation' (BP), 'chemokine activity' (MF), and 'condensed chromosome (CC)'. The KEGG pathway was mainly enriched in 'cell cycle', 'progesterone-mediated oocyte maturation', 'chemokine signaling pathway', 'IL-17 signaling pathway', 'legionellosis', and 'rheumatoid arthritis'. DNA topoisomerase II- $\alpha$ (TOP2A), mitotic arrest deficient 2 like 1 (MAD2L1), cyclin B1 (CCNB1), checkpoint kinase 1 (CHEK1), cell division cycle 6 (CDC6) and ubiquitin conjugating enzyme E2 C (UBE2C) were indicated as hub genes. Furthermore, survival analysis revealed that TOP2A, MAD2L1, CDC6 and CHEK1 may serve as prognostic biomarkers in CRC. The present study provided insights into the molecular mechanism of CRC that may be useful in further investigations.

\section{Introduction}

Colorectal cancer (CRC) is a global burden ranking third in terms of incidence and second in terms of mortality, with $>1.8$ million new CRC cases and 881,000 estimated deaths in 2018 (1). The main reason for the poor 5 -year overall survival in CRC is late detection, when the opportunity for treatment has passed. Despite progress in novel therapies, early detection remains a challenge (2). A variety of tests are available for screening and detecting CRC; all, however, have their disadvantages (3-5). It is therefore urgent to identify novel diagnostic and prognostic biomarkers for CRC.

In recent years, high-quality microarray and highthroughput sequencing have been effective in detecting the development and progression of CRC, and even in screening biomarkers for CRC diagnosis, therapy and prognosis.

A number of gene profiles can be obtained from public databases such as Gene Expression Omnibus (GEO) and The Cancer Genome Atlas (TCGA), both of which can expand our understanding of cancer. Limitations and inconsistent results may exist, due to different microarray platforms and small sample sizes, but integrated bioinformatics methods may overcome these limitations. 
Several recent studies have identified certain key genes and pathways in CRC using bioinformatics analysis $(6,7)$. Based on these articles, updated datasets were selected and a gene classification method (clusterProfiler package in R) was used for Gene Ontology (GO) and Kyoto Encyclopedia of Genes and Genomes (KEGG) enrichment analysis; the prognostic role of hub genes was then ascertained using the TCGA dataset. In the present study, integrated analysis was first performed to identify the common DEGs from multiple microarrays (GSE113513, GSE9348 and GSE22598) and TCGA CRC RNA-seq data. Next, GO and KEGG enrichment analysis was conducted to identify the potential biological role of DEGs. A protein-protein interaction (PPI) network created based on the Search Tool for the Retrieval of Interacting Genes/Proteins (STRING; version 10.5; http://string-db.org/) database, and the significant modules and hub genes were then selected from the network. The prognostic roles of hub genes were analyzed using TCGA.

\section{Materials and methods}

Gene expression profile data. The raw microarray data of CRC [GSE9348 (8), GSE22598 (9), GSE113513 (unpublished, 2018)] were downloaded from the GEO database (10) (Table I). All datasets fulfilled the following criteria: i) Human CRC tissue samples were used for the profiles; ii) normal samples were matched to the tumor tissues, when data could not be matched, matching was based on patient information, such as age and sex; and iii) The sample size per dataset was $>10$. The level 3 RNA sequencing data of CRC and normal samples as well as the CRC clinical information were downloaded from the Genomic Data Commons (GDC) which were retrieved from TCGA (https://tcga-data.nci.nih. gov/tcga/) database.

Data pre-processing and DEG identification. CEL files from three Affymetrix microarrays were downloaded from GEO, and pre-processed using the Affy package (version 1.60.0; https://bioconductor.org/packages/release/bioc/html/affy.

html) in R software (version 3.4.3; http://www.r-project. org/). The Robust Multi-array Average (RMA) method (11) was used for the pre-processing, which included background correcting, normalizing and calculating expression. The latest annotation files were downloaded for re-annotation. The Limma package (version 3.34.8) (12) in R software was subsequently used to screen DEGs between CRC and matched normal tissues in the microarray. The RNA sequencing data were obtained from TCGA and Ensembl Release 93 (http://jul2018.archive.ensembl.org/index.html) files were used for annotation and all the data processing and normalization were finished using the Perl (version 5.28.2; https://www.perl.org/) and R scripts. The edgeR package (version 3.24.3) (13) was used for DEG screening and the trimmed mean of M-values normalization method in edgeR was used to normalize the raw data. Notably, $\log _{2} \mathrm{FCl}>1$, $\mathrm{P}<0.05$ and adjusted $\mathrm{P}<0.05$ were considered the cut-off criteria. Intersect function was used to identify the common DEGs, and a Venn diagram was created using Venny (version 2.1) (14). All common DEGs in these datasets were selected for further study.
$G O$ and KEGG pathway analyses. To elucidate the potential gene functional annotation and pathway enrichment associated with the common DEGs. GO $(15,16)$ and KEGG $(17-19)$ analyses were performed using the clusterProfiler (version 3.10.1) package (20). The enrichplot and DOSE (21) packages were used to supply enrichment result visualization to help interpretation. $\mathrm{P}<0.05$ and adjusted $\mathrm{P}<0.05$ were set as the threshold values.

PPI network construction and module selection. The online STRING database was used to identify potential interaction among the common DEGs, and a confidence score of $\geq 0.4$ was set as the threshold. Cytoscape (version 3.6.1) (22) was used to visualize the PPI network of common DEGs. The MCODE plug-in (23) was used to search sub-networks of the PPI network and the default parameters (Degree cutoff $\geq 10$, node score cutoff $\geq 0.4, \mathrm{~K}$-core $\geq 4$, and $\max$ depth $=100$.) were set in the functional interface of Cytoscape software. GO and KEGG enrichment analyses of cluster modules were performed using ClueGO plug-in (24) with default parameters. The Cytohubba plug-in (25) was used to explore hub genes, and the top ten were generated using Maximal Clique Centrality (MCC), closeness and degree methods. The intersect function was used to identify the common hub genes.

Survival analysis of hub genes. In order to identify the potential prognostic role of hub genes, Kaplan-Meier analysis was performed based on the expression and clinical data of TCGA, and Perl was used to merge data. Hub genes were divided into two strata based on expression level and median value. The survival (version 2.44-1.1; https://cran.r-project. org/web/packages/survival/index.html) and survminer package (version 0.4.3; https://cran.r-project.org/web/packages/survminer/index.html) in $\mathrm{R}$ were used.

\section{Results}

Identification of DEGs in GEO and TCGA. Following pre-processing of the raw data, DEGs were identified by $\mathrm{R}$ package. In total, 931 DEGs (543 down- and 388 upregulated genes), 1,420 DEGs (759 down- and 661 upregulated genes), 1,324 DEGs (659 down- and 665 upregulated genes) and 12,237 DEGs (3,776 down- and 8,461 upregulated genes) were screened from the GSE113513, GSE9348, GSE22598 and TCGA datasets, respectively. Heatmaps of DEGs are presented in Fig. S1, and volcano plots in Fig. S2. The intersect function revealed 405 common DEGs, including 236 downand 169 upregulated DEGs from four independent datasets (Fig. 1). The top 20 down- and upregulated DEGs from the four datasets are listed in Table II.

GO and KEGG enrichment analysis. To explore the potential biological function of common DEGs, GO and KEGG pathways enrichment analyses were conducted using the ClusterProfiler package. In the present study, downregulated DEGs were mainly enriched in 'detoxification of copper ion' (BP), 'oxidoreductase activity, acting on $\mathrm{CH}-\mathrm{OH}$ group of donors, NAD or NADP as acceptor' (MF) and 'brush border' (CC) respectively. Upregulated DEGs were mainly involved in 'nuclear division' (BP), 'snoRNA binding' (MF) and 'nucleolar part' (CC).'The 
Table I. Information on the datasets included in the current study.

\begin{tabular}{|c|c|c|c|}
\hline Dataset & Reference & Platform & $\begin{array}{l}\text { No. of sample } \\
\text { (normal/tumo }\end{array}$ \\
\hline GSE9348 & Hong et al, 2010 (8) & $\begin{array}{l}\text { [HG-U133_Plus_2] Affymetrix Human } \\
\text { Genome U133 Plus 2.0 Array }\end{array}$ & $12 / 12$ \\
\hline GSE22598 & Okazaki et al, 2011 (9) & $\begin{array}{l}\text { [HG-U133_Plus_2] Affymetrix Human } \\
\text { Genome U133 Plus 2.0 Array }\end{array}$ & $17 / 17$ \\
\hline GSE113513 & Peng et al, 2018 (unpublished) & $\begin{array}{l}\text { [PrimeView] Affymetrix Human } \\
\text { Gene Expression Array }\end{array}$ & $14 / 14$ \\
\hline TCGA_CRC & The Cancer Genome Atlas (TCGA) data portal & IlluminaHiseq (Illumina, San Diego, CA) & $51 / 647$ \\
\hline
\end{tabular}
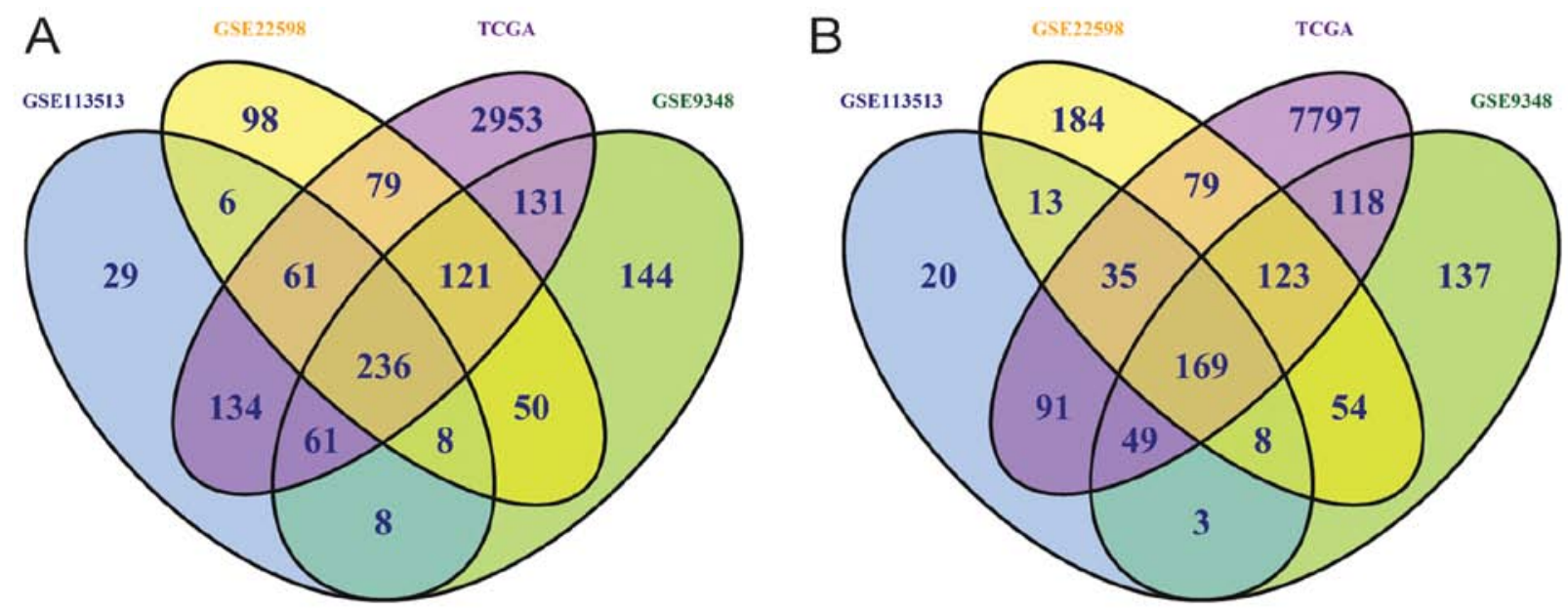

Figure 1. Intersected analysis was used for the identification of 405 simultaneously changed DEGs from four datasets (GSE113513, GSE9348, GSE22598 and TCGA). (A) A total of 236 downregulated and (B) 169 upregulated overlapping DEGs was identified. Different colored regions represent different datasets, and the intersective area denotes the simultaneously changed DEGs. DEGs, differentially expressed genes; TCGA, The Cancer Genome Atlas.

GO distribution of down- and upregulated DEGs is presented in Fig. 2A and B and details of the top $15 \mathrm{GO}$ terms in Table III.

According to KEGG enrichment analysis, four significant pathways of common DEGs were identified, including: 'Mineral absorption', 'nitrogen metabolism', 'cell cycle' and 'caffeine metabolism' (Fig. 2C). The significant genes in these pathways are presented in Fig. 2D.

PPI network and module selection. The PPI network of DEGs was constructed with 268 nodes and 1,027 edges (Fig. 3A). The Degree, MCC and Closeness methods were performed to calculate the top 10 ranking hub genes. The results revealed 6 genes identified as hub genes, including DNA topoisomerase II- $\alpha$ (TOP2A), mitotic arrest deficient 2 like 1 (MAD2L1), cyclin B1 (CCNB1), checkpoint kinase 1 (CHEK1), cell division cycle 6 (CDC6), ubiquitin conjugating enzyme E2 C (UBE2C) (Fig. 3B). MCODE was used to identify the significant cluster modules in the PPI network and the top module was selected (Fig. 3C). Following GO annotation screening, the module (44 nodes and 462 edges) was revealed to be associated with 'sister chromatid segregation' (BP), 'chemokine activity' (MF), 'condensed chromosome (CC)' (Table IV) and KEGG pathway enrichment analysis revealed that the top module was mainly enriched in 'cell cycle', 'progesterone-mediated oocyte maturation', 'chemokine signaling pathway', 'IL-17 signaling pathway', 'legionellosis' and 'rheumatoid arthritis'; related information were represent in Table V.

Survival analysis. The prognostic role of hub genes was analyzed using Kaplan-Meier method based on the TCGA data. Among these hub genes, a low expression of CDC6, CHEK1, MAD2L1 and TOP2A was associated with poor prognosis in CRC patients (Fig. 4).

\section{Discussion}

In the present study, three GEOs and TCGA data were integrated, and non-paired GEO data were manually matched to increase accuracy and stabilization. In total, 236 down- and 169 upregulated DEGs were identified. The common downregulated DEGs were mostly enriched in 'detoxification of copper ion' (BP), 'oxidoreductase activity, acting on $\mathrm{CH}-\mathrm{OH}$ group of donors, NAD or NADP as acceptor' (MF) and 'brush border' (CC), and the upregulated DEGs were mainly associated with 'nuclear division' (BP), 'snoRNA binding' (MF) and 'nucleolar part' (CC). Moreover, KEGG enrichment analysis identified that the common DEGs were largely 
Table II. Top 20 down- and upregulated overlapping DEGs in GSE113513, GSE9348, GSE22598 and TCGA were screened by intersected analysis.

\begin{tabular}{|c|c|c|c|c|c|}
\hline Genes & GSE113513 & GSE9348 & GSE22598 & TCGA & Regulation \\
\hline AQP8 & -4.00 & -6.74 & -2.75 & -6.87 & Down \\
\hline CLCA4 & -2.30 & -7.61 & -4.91 & -5.35 & Down \\
\hline GUCA2B & -4.82 & -5.08 & -3.37 & -6.06 & Down \\
\hline MS4A12 & -3.68 & -6.04 & -3.54 & -5.36 & Down \\
\hline GUCA2A & -4.10 & -5.00 & -3.72 & -5.11 & Down \\
\hline CA2 & -3.68 & -5.32 & -3.76 & -4.81 & Down \\
\hline ABCG2 & -2.39 & -6.03 & -3.93 & -4.68 & Down \\
\hline CLDN8 & -3.69 & -4.40 & -3.65 & -5.07 & Down \\
\hline GCG & -4.57 & -3.92 & -4.15 & -4.13 & Down \\
\hline ZG16 & -3.18 & -4.70 & -4.45 & -4.44 & Down \\
\hline PKIB & -3.21 & -4.99 & -3.85 & -3.95 & Down \\
\hline CA4 & -3.39 & -4.54 & -3.07 & -4.76 & Down \\
\hline BEST4 & -3.50 & -2.72 & -3.66 & -5.85 & Down \\
\hline CA1 & -3.33 & -3.20 & -1.94 & -6.51 & Down \\
\hline MT1M & -3.29 & -3.90 & -3.35 & -4.30 & Down \\
\hline CD177 & -2.84 & -4.29 & -2.35 & -5.22 & Down \\
\hline HSD17B2 & -2.63 & -5.14 & -3.04 & -3.39 & Down \\
\hline INSL5 & -3.31 & -2.03 & -3.00 & -5.82 & Down \\
\hline $\mathrm{ADH} 1 \mathrm{C}$ & -3.26 & -3.50 & -3.10 & -4.01 & Down \\
\hline CLCA1 & -3.45 & -3.77 & -3.62 & -2.54 & Down \\
\hline FOXQ1 & 4.47 & 5.16 & 5.55 & 6.47 & Up \\
\hline KRT23 & 3.69 & 4.25 & 4.28 & 7.37 & Up \\
\hline LY6G6D & 3.50 & 4.09 & 3.63 & 5.42 & $\mathrm{Up}$ \\
\hline MMP7 & 3.15 & 3.11 & 2.38 & 7.03 & $\mathrm{Up}$ \\
\hline $\mathrm{CDH} 3$ & 2.77 & 2.31 & 2.76 & 5.77 & Up \\
\hline MMP3 & 2.91 & 2.94 & 2.77 & 4.83 & Up \\
\hline CST1 & 1.25 & 2.42 & 1.00 & 8.33 & Up \\
\hline CRNDE & 3.17 & 2.36 & 2.73 & 4.60 & Up \\
\hline DPEP1 & 2.97 & 3.06 & 4.14 & 2.61 & Up \\
\hline MMP1 & 2.46 & 2.59 & 3.14 & 4.56 & $\mathrm{Up}$ \\
\hline EPHX4 & 3.01 & 2.32 & 2.95 & 4.43 & $\mathrm{Up}$ \\
\hline CTHRC1 & 1.21 & 4.47 & 3.15 & 3.78 & Up \\
\hline CLDN1 & 2.18 & 2.81 & 2.74 & 4.84 & $\mathrm{Up}$ \\
\hline CEL & 1.56 & 2.53 & 2.37 & 6.10 & Up \\
\hline CLDN2 & 1.54 & 2.57 & 2.75 & 5.57 & Up \\
\hline SLC35D3 & 1.79 & 2.97 & 3.24 & 4.35 & $\mathrm{Up}$ \\
\hline COL11A1 & 1.59 & 2.49 & 1.93 & 6.33 & $\mathrm{Up}$ \\
\hline CXCL3 & 3.22 & 3.60 & 2.34 & 2.92 & Up \\
\hline SLCO1B3 & 1.43 & 1.64 & 2.55 & 6.44 & Up \\
\hline CKMT2 & 3.33 & 2.55 & 2.68 & 3.46 & $\mathrm{Up}$ \\
\hline
\end{tabular}

Each column represents a dataset and each row a gene. The values in the table represent the $\log _{2} \mathrm{FC}$ in the datasets. DEGs, differentially expressed genes; TCGA, The Cancer Genome Atlas; FC, fold change.

involved in 'mineral absorption', 'nitrogen metabolism', 'cell cycle' and 'caffeine metabolism'. Investigating these significant pathways may promote the understanding of CRC development.

Minerals are one of the five fundamental groups of nutrients; regular mineral absorption plays a vital role in sustaining life. Deficiency and insufficiency of minerals may be associated with and increase the risk of cancer; for example, efficient absorption of vitamin D may prevent CRC (26). The cell cycle is controlled by various mechanisms, which ensure correct cell division; loss of normal cell cycle control is a hallmark of cancer (27). An increasing number of studies 

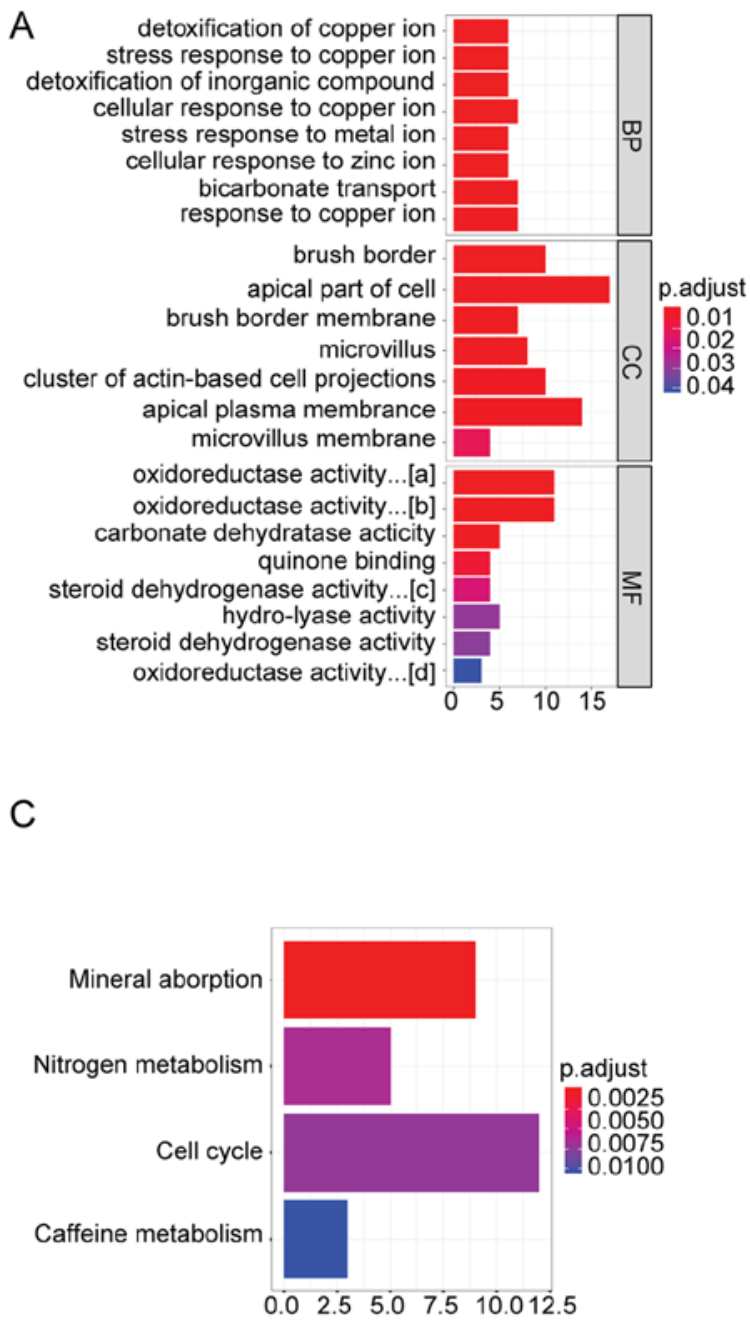
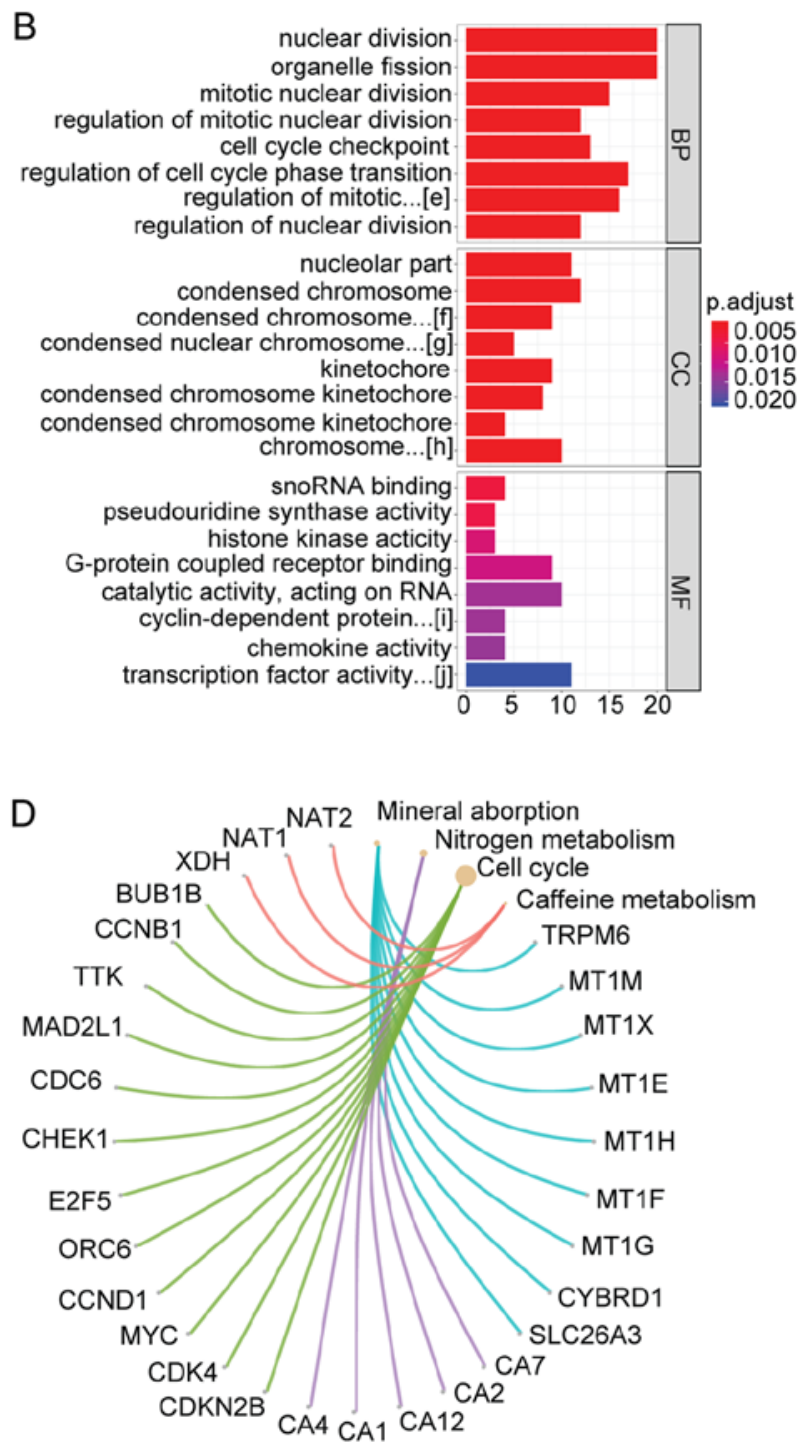

Figure 2. GO and KEGG enrichment analysis of the overlapping DEGs. (A and B) Top significantly enriched GO terms of down- and upregulated DEGs, including $\mathrm{BP}, \mathrm{CC}$ and MF. The $\mathrm{x}$-axis represents the number of DEGs involved in GO terms, and the y-axis the significantly enriched GO terms. (C) KEGG pathway enrichment analysis of overlapping DEGs. The X-axis indicates the number of DEGs involved in the significant KEGG pathway, and the $\mathrm{y}$-axis the terms of the significant KEGG pathway. (D) DEGs associated with the significant KEGG pathway. GO, gene ontology; KEGG, Kyoto Encyclopedia of Genes and Genomes; DEGs, differentially expressed genes. (a) Oxidoreductase activity, acting on the CH-OH group of donors, NAD or NADP as acceptor; (b) oxidoreductase activity, acting on $\mathrm{CH}-\mathrm{OH}$ group of donors; (c) steroid dehydrogenase activity, acting on the CH-OH group of donors, NAD or NADP as acceptor; (d) oxidoreductase activity, acting on the $\mathrm{CH}-\mathrm{NH} 2$ group of donors, oxygen as acceptor; (e) regulation of mitotic cell cycle phase transition; (f) condensed chromosome, centromeric region; (g) condensed nuclear chromosome, centromeric region; (h) chromosome, centromeric region; (i) cyclin-dependent protein serine/threonine kinase regulator activity; (j) transcription factor activity, RNA polymerase II proximal promoter sequence-specific DNA binding.

has revealed that targeting the deregulation of the cell cycle in cancer is a potential therapeutic strategy (28). Therefore, investigating the cell cycle pathway may promote the understanding of carcinogenic mechanisms and insights into CRC treatment options. Nitrogen is an essential component of life that is involved in processes of both proteins and nucleic acids. Aberrant expression of nitrogen species could affect the risk of cancer (29). Certain studies have reported that caffeine could decrease the risk of CRC (30), inhibit colon cancer cell proliferation $(31,32)$. Metabolic profiling revealed that caffeine metabolism differed significantly between colorectal adenoma polyps and CRC patients which may influence CRC development and outcome (33). Investigating these significant pathways may elucidate the mechanism of CRC progression.
Recently, certain important biomarkers, including CCL19, CXCL1, CXCL5, CXCL11, CXCL12, GNG4, INSL5, NMU, PYY and SST, were identified using integrated bioinformatics analysis. Furthermore, a prognostic gene signature consisting of 9 genes was also identified (34). In the present study, data from three GEO datasets (GSE22598, GSE113513 and GSE9348) and TCGA were combined for screening stable DEGs, then different calculation methods and intersect function analysis were used, and potential biomarkers were revealed that had not been previously screened out. A PPI network was constructed with DEGs, and then 6 hub genes (TOP2A, MAD2L1, CCNB1, CHEK1, CDC6 and UBE2C) were identified. Following survival analysis based on the TCGA data, the low expression of CDC6, CHEK1, MAD2L1 and TOP2A was revealed to be associated with poor prognosis. 
Table III. GO analysis of down- and upregulated overlapping DEGs associated with CRC.

\begin{tabular}{|c|c|c|c|c|c|}
\hline Expression & Terms & ID & Description & q-value & Genes \\
\hline \multirow[t]{15}{*}{ Upregulated } & $\mathrm{BP}$ & GO:0000280 & Nuclear division & $4.33 \times 10^{-7}$ & $\begin{array}{l}\text { RRS1/CKS2/TRIP13/CCND1/FIGNL1/TPX2/ } \\
\text { ANLN/UBE2C/CHEK1/NUF2/RAD54B/ } \\
\text { DLGAP5/CDC6/MAD2L1/TOP2A/TTK/ } \\
\text { CCNB1/ASPM/BUB1B/AURKA }\end{array}$ \\
\hline & $\mathrm{BP}$ & GO:0048285 & Organelle fission & $1.16 \times 10^{-6}$ & $\begin{array}{l}\text { RRS1/CKS2/TRIP13/CCND1/FIGNL1/TPX2/ } \\
\text { ANLN/UBE2C/CHEK1/NUF2/RAD54B/ } \\
\text { DLGAP5/CDC6/MAD2L1/TOP2A/TTK/ } \\
\text { CCNB1/ASPM/BUB1B/AURKA }\end{array}$ \\
\hline & $\mathrm{BP}$ & GO:0140014 & Mitotic nuclear division & $6.51 \times 10^{-6}$ & $\begin{array}{l}\text { RRS1/TRIP13/CCND1/TPX2/ANLN/ } \\
\text { UBE2C/CHEK1/NUF2/DLGAP5/CDC6/ } \\
\text { MAD2L1/TTK/CCNB1/BUB1B/AURKA }\end{array}$ \\
\hline & BP & GO:0007088 & $\begin{array}{l}\text { Regulation of mitotic } \\
\text { nuclear division }\end{array}$ & $1.18 \times 10^{-5}$ & $\begin{array}{l}\text { TRIP13/CCND1/ANLN/UBE2C/CHEK1/ } \\
\text { DLGAP5/CDC6/MAD2L1/TTK/CCNB1/ } \\
\text { BUB1B/AURKA }\end{array}$ \\
\hline & $\mathrm{BP}$ & GO:0000075 & Cell cycle checkpoint & $1.18 \times 10^{-5}$ & $\begin{array}{l}\text { TRIP13/CCND1/ARID3A/CHEK1/CDC6/ } \\
\text { MAD2L1/TOP2A/TTK/CCNB1/SOX4/ } \\
\text { BUB1B/AURKA/PROX1 }\end{array}$ \\
\hline & $\mathrm{CC}$ & GO:0044452 & Nucleolar part & $4.66 \times 10^{-5}$ & $\begin{array}{l}\text { TAF1D/DKC1/UTP4/WDR43/NOP58/RRS1/ } \\
\text { RPP40/NUFIP1/ORC6/POLR1D/E2F5 }\end{array}$ \\
\hline & $\mathrm{CC}$ & GO:0000793 & Condensed chromosome & $4.66 \times 10^{-5}$ & $\begin{array}{l}\text { RRS1/SKA3/CHEK1/NUF2/CENPA/ } \\
\text { MAD2L1/TOP2A/CCNB1/SPC25/ERCC6L/ } \\
\text { BUB1B/AURKA }\end{array}$ \\
\hline & $\mathrm{CC}$ & GO:0000779 & $\begin{array}{l}\text { Condensed chromosome, } \\
\text { centromeric region }\end{array}$ & $5.94 \times 10^{-5}$ & $\begin{array}{l}\text { SKA3/NUF2/CENPA/MAD2L1/CCNB1/ } \\
\text { SPC25/ERCC6L/BUB1B/AURKA }\end{array}$ \\
\hline & $\mathrm{CC}$ & GO:0000780 & $\begin{array}{l}\text { Condensed nuclear } \\
\text { Chromosome, } \\
\text { centromeric region }\end{array}$ & $1.13 \times 10^{-4}$ & NUF2/CENPA/CCNB1/BUB1B/AURKA \\
\hline & $\mathrm{CC}$ & GO:0000776 & Kinetochore & $1.13 \times 10^{-4}$ & $\begin{array}{l}\text { SKA3/NUF2/CENPA/MAD2L1/TTK/ } \\
\text { CCNB1/SPC25/ERCC6L/BUB1B }\end{array}$ \\
\hline & MF & GO:0030515 & snoRNA binding & $2.99 \times 10^{-3}$ & DKC1/NOP58/DDX21/NUFIP1 \\
\hline & MF & GO:0009982 & $\begin{array}{l}\text { Pseudouridine synthase } \\
\text { activity }\end{array}$ & $3.70 \times 10^{-3}$ & PUS1/PUS7/DKC1 \\
\hline & MF & GO:0035173 & Histone kinase activity & $7.05 \times 10^{-3}$ & CHEK1/CCNB1/AURKA \\
\hline & MF & GO:0001664 & $\begin{array}{l}\text { G protein-coupled } \\
\text { receptor binding }\end{array}$ & $8.09 \times 10^{-3}$ & $\begin{array}{l}\text { CXCL3/RNF43/HOMER1/NMU/CXCL8/ } \\
\text { ZNRF3/CXCL1/CTHRC1/CXCL5 }\end{array}$ \\
\hline & MF & GO:0140098 & $\begin{array}{l}\text { Catalytic activity, acting } \\
\text { on RNA }\end{array}$ & $1.15 \times 10^{-2}$ & $\begin{array}{l}\text { NOB1/PUS1/EXOSC5/NOP2/RPP40/ } \\
\text { DDX21/RNASEH2A/POLR1D/RAD54B/ } \\
\text { AZGP1 }\end{array}$ \\
\hline \multirow[t]{6}{*}{ Downregulated } & $\mathrm{BP}$ & GO:0010273 & $\begin{array}{l}\text { Detoxification of } \\
\text { copper ion }\end{array}$ & $1.22 \times 10^{-5}$ & MT1M/MT1X/MT1E/MT1H/MT1F/MT1G \\
\hline & $\mathrm{BP}$ & GO:1990169 & $\begin{array}{l}\text { Stress response to } \\
\text { copper ion }\end{array}$ & $1.22 \times 10^{-5}$ & MT1M/MT1X/MT1E/MT1H/MT1F/MT1G \\
\hline & $\mathrm{BP}$ & GO:0061687 & $\begin{array}{l}\text { Detoxification of } \\
\text { inorganic compound }\end{array}$ & $1.22 \times 10^{-5}$ & MT1M/MT1X/MT1E/MT1H/MT1F/MT1G \\
\hline & $\mathrm{BP}$ & GO:0071280 & $\begin{array}{l}\text { Cellular response to } \\
\text { copper ion }\end{array}$ & $1.22 \times 10^{-5}$ & $\begin{array}{l}\text { MT1M/MT1X/MT1E/MT1H/MT1F/MT1G/ } \\
\text { AOC1 }\end{array}$ \\
\hline & $\mathrm{BP}$ & GO:0097501 & $\begin{array}{l}\text { Stress response to } \\
\text { metal ion }\end{array}$ & $1.35 \times 10^{-5}$ & MT1M/MT1X/MT1E/MT1H/MT1F/MT1G \\
\hline & $\mathrm{CC}$ & GO:0005903 & Brush border & $6.96 \times 10^{-5}$ & $\begin{array}{l}\text { CDHR5/TRPM6/SCIN/CDHR2/CA4/LIMA1/ } \\
\text { CYBRD1/MYO1A/SLC26A3/SI }\end{array}$ \\
\hline
\end{tabular}


Table III. Continued.

\begin{tabular}{|c|c|c|c|c|c|}
\hline Expression & Terms & ID & Description & q-value & Genes \\
\hline & $\mathrm{CC}$ & GO:0045177 & Apical part of cell & $3.15 \times 10^{-4}$ & $\begin{array}{l}\text { ABCG2/CA2/RAB27A/CDHR5/PTPRH/ } \\
\text { TRPM6/AQP8/CDHR2/CA4/SCNN1B/ } \\
\text { CLCA4/CEACAM1/FABP1/CEACAM7/ } \\
\text { MYO1A/SLC26A3/SI }\end{array}$ \\
\hline & $\mathrm{CC}$ & GO:0031526 & Brush border membrane & $3.15 \times 10^{-4}$ & $\begin{array}{l}\text { CDHR5/TRPM6/CDHR2/CA4/LIMA1/ } \\
\text { CYBRD1/SLC26A3 }\end{array}$ \\
\hline & $\mathrm{CC}$ & GO:0005902 & Microvillus & $6.78 \times 10^{-4}$ & $\begin{array}{l}\text { CA2/CDHR5/PTPRH/CDHR2/CEACAM1/ } \\
\text { CLCA1/AOC3/MYO1A }\end{array}$ \\
\hline & $\mathrm{CC}$ & GO:0098862 & $\begin{array}{l}\text { Cluster of actin-based } \\
\text { cell projections }\end{array}$ & $8.31 \times 10^{-4}$ & $\begin{array}{l}\text { CDHR5/TRPM6/SCIN/CDHR2/CA4/LIMA1/ } \\
\text { CYBRD1/MYO1A/SLC26A3/SI }\end{array}$ \\
\hline & MF & GO:0016616 & $\begin{array}{l}\text { Oxidoreductase activity, } \\
\text { acting on the } \mathrm{CH}-\mathrm{OH} \\
\text { group of donors, NAD } \\
\text { or NADP as acceptor }\end{array}$ & $6.48 \times 10^{-5}$ & $\begin{array}{l}\text { UGDH/ADH1B/HPGD/LDHD/ADH1C/ } \\
\text { DHRS11/DHRS9/HSD17B2/AKR1B10/ } \\
\text { HSD11B2/BMP2 }\end{array}$ \\
\hline & MF & GO:0016614 & $\begin{array}{l}\text { Oxidoreductase activity, } \\
\text { acting on } \mathrm{CH}-\mathrm{OH} \text { group } \\
\text { of donors }\end{array}$ & $1.09 \times 10^{-4}$ & $\begin{array}{l}\text { UGDH/ADH1B/HPGD/LDHD/ADH1C/ } \\
\text { DHRS11/DHRS9/HSD17B2/AKR1B10/ } \\
\text { HSD11B2/BMP2 }\end{array}$ \\
\hline & MF & GO:0004089 & $\begin{array}{l}\text { Carbonate dehydratase } \\
\text { activity }\end{array}$ & $1.26 \times 10^{-4}$ & $\mathrm{CA} 7 / \mathrm{CA} 2 / \mathrm{CA} 12 / \mathrm{CA} 1 / \mathrm{CA} 4$ \\
\hline & MF & GO:0048038 & Quinone binding & $4.90 \times 10^{-3}$ & ETFDH/TP53I3/AOC3/AOC1 \\
\hline & MF & GO:0033764 & $\begin{array}{l}\text { Steroid dehydrogenase } \\
\text { activity, acting on the } \\
\mathrm{CH}-\mathrm{OH} \text { group of donors, } \\
\text { NAD or NADP as acceptor }\end{array}$ & $1.52 \times 10^{-2}$ & DHRS11/DHRS9/HSD17B2/HSD11B2 \\
\hline
\end{tabular}

Adjusted $\mathrm{P}<0.05$ was considered to be significant. DEGs, differentially expressed genes; CRC, colorectal cancer; q-value, adjusted P-value; $\mathrm{GO}$, Gene Ontology; BP, biological process; CC, cellular component; MF, molecular function.

A

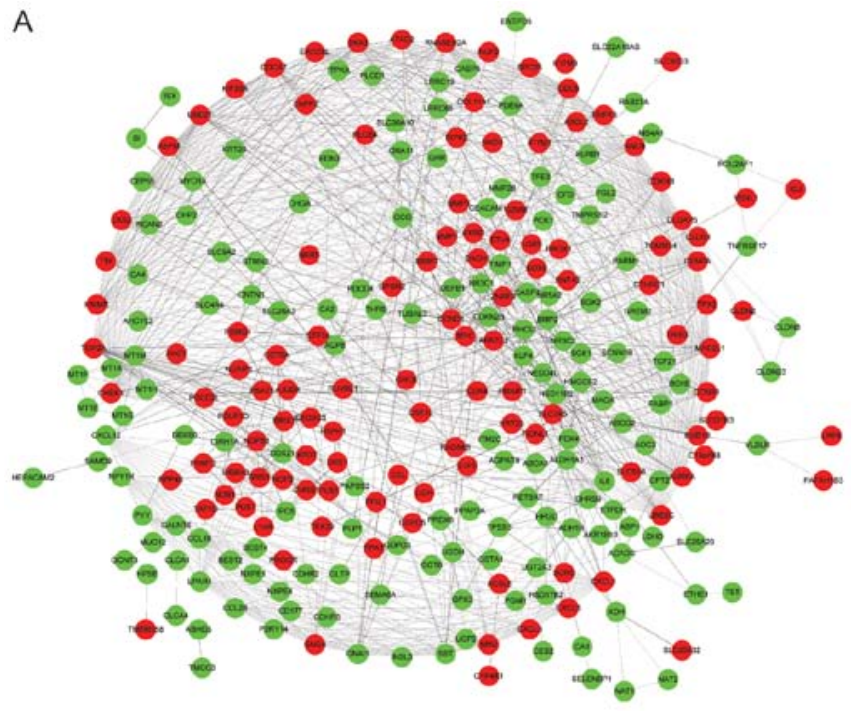

B

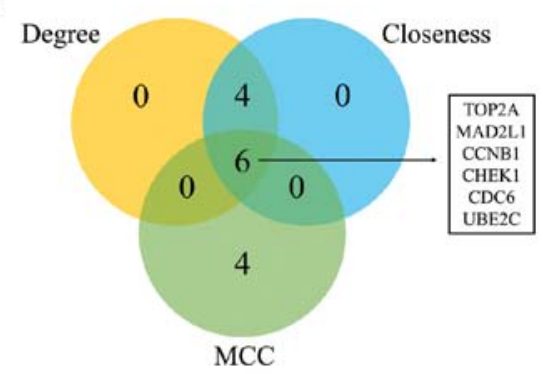

C
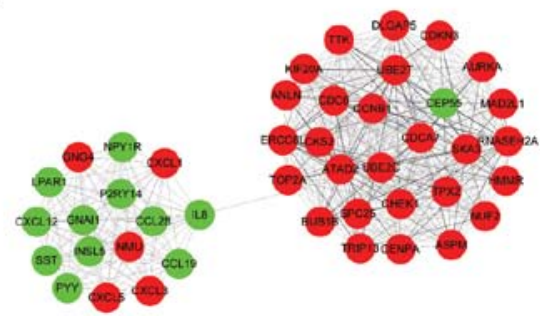

Figure 3. PPI network and hub clustering modules. (A) PPI networks of the overlapping DEGs. (B) Hub genes were screened from the PPI network using the Closeness, Degree and MCC methods. (C) The top module was selected from the PPI network. Red circles indicate upregulated genes and green circles downregulated genes. PPI, protein-protein interaction; DEGs, differentially expressed genes.

TOP2A was an important nuclear enzyme involved in DNA transcription and replication. Aberrant TOP2A expression has been identified in several types of cancer, such as CRC, breast carcinomas, gastric cancer and bladder carcinomas (35-38). In 
Table IV. GO analysis of selected module-associated DEGs.

\begin{tabular}{|c|c|c|c|c|}
\hline ID & GO Terms & Ontology & q-value & Genes \\
\hline GO:0000819 & Sister chromatid segregation & $\mathrm{BP}$ & $5.54 \times 10^{-13}$ & $\begin{array}{l}\text { BUB1B/CCNB1/CDC6/CENPA/DLGAP5/ } \\
\text { ERCC6L/MAD2L1/NUF2/SPC25/TOP2A/ } \\
\text { TRIP13/TTK/UBE2C }\end{array}$ \\
\hline GO:0007088 & $\begin{array}{l}\text { Regulation of mitotic nuclear } \\
\text { division }\end{array}$ & $\mathrm{BP}$ & $8.77 \times 10^{-12}$ & $\begin{array}{l}\text { ANLN/AURKA/BUB1B/CCNB1/CDC6/ } \\
\text { CHEK1/DLGAP5/MAD2L1/TRIP13/TTK/ } \\
\text { UBE2C }\end{array}$ \\
\hline GO:0098813 & $\begin{array}{l}\text { Nuclear chromosome } \\
\text { segregation }\end{array}$ & $\mathrm{BP}$ & $1.24 \times 10^{-11}$ & $\begin{array}{l}\text { BUB1B/CCNB1/CDC6/CENPA/DLGAP5/ } \\
\text { ERCC6L/MAD2L1/NUF2/SPC25/TOP2A/ } \\
\text { TRIP13/TTK/UBE2C }\end{array}$ \\
\hline GO:0051304 & Chromosome separation & $\mathrm{BP}$ & $1.37 \times 10^{-11}$ & $\begin{array}{l}\text { BUB1B/CCNB1/CDC6/DLGAP5/MAD2L1/ } \\
\text { TOP2A/TRIP13/TTK/UBE2C }\end{array}$ \\
\hline GO:0030071 & $\begin{array}{l}\text { Regulation of mitotic } \\
\text { metaphase/anaphase } \\
\text { transition }\end{array}$ & $\mathrm{BP}$ & $1.46 \times 10^{-11}$ & $\begin{array}{l}\text { BUB1B/CCNB1/CDC6/DLGAP5/MAD2L1/ } \\
\text { TRIP13/TTK/UBE2C }\end{array}$ \\
\hline GO:0000793 & Condensed chromosome & $\mathrm{CC}$ & $2.53 \times 10^{-10}$ & $\begin{array}{l}\text { AURKA/BUB1B/CCNB1/CENPA/CHEK1/ } \\
\text { ERCC6L/MAD2L1/NUF2/SKA3/SPC25/ } \\
\text { TOP2A }\end{array}$ \\
\hline GO:0000779 & $\begin{array}{l}\text { Condensed chromosome/ } \\
\text { centromeric region }\end{array}$ & $\mathrm{CC}$ & $5.40 \times 10^{-10}$ & $\begin{array}{l}\text { AURKA/BUB1B/CCNB1/CENPA/ERCC6L/ } \\
\text { MAD2L1/NUF2/SKA3/SPC25 }\end{array}$ \\
\hline GO:0000775 & $\begin{array}{l}\text { Chromosome/centromeric } \\
\text { region }\end{array}$ & $\mathrm{CC}$ & $1.59 \times 10^{-9}$ & $\begin{array}{l}\text { AURKA/BUB1B/CCNB1/CENPA/ERCC6L/ } \\
\text { MAD2L1/NUF2/SKA3/SPC25/TTK }\end{array}$ \\
\hline GO:0000776 & Kinetochore & $\mathrm{CC}$ & $1.80 \times 10^{-9}$ & $\begin{array}{l}\text { BUB1B/CCNB1/CENPA/ERCC6L/MAD2L1/ } \\
\text { NUF2/SKA3/SPC25/TTK }\end{array}$ \\
\hline GO:0000777 & $\begin{array}{l}\text { Condensed chromosome } \\
\text { kinetochore }\end{array}$ & $\mathrm{CC}$ & $8.17 \times 10^{-9}$ & $\begin{array}{l}\text { BUB1B/CCNB1/CENPA/ERCC6L/MAD2L1/ } \\
\text { NUF2/SKA3/SPC25 }\end{array}$ \\
\hline GO:0008009 & Chemokine activity & MF & $1.41 \times 10^{-9}$ & $\begin{array}{l}\text { CCL19/CCL28/CXCL1/CXCL12/CXCL3/ } \\
\text { CXCL5/CXCL8 }\end{array}$ \\
\hline GO:0042379 & Chemokine receptor binding & MF & $8.09 \times 10^{-9}$ & $\begin{array}{l}\text { CCL19/CCL28/CXCL1/CXCL12/CXCL3/ } \\
\text { CXCL5/CXCL8 }\end{array}$ \\
\hline GO:0045236 & $\begin{array}{l}\text { CXCR chemokine receptor } \\
\text { binding }\end{array}$ & MF & $1.46 \times 10^{-8}$ & CXCL1/CXCL12/CXCL3/CXCL5/CXCL8 \\
\hline GO:0035173 & Histone kinase activity & MF & $1.99 \times 10^{-4}$ & AURKA/CCNB1/CHEK1 \\
\hline
\end{tabular}

Adjusted $\mathrm{P}<0.05$ was considered as significanct. DEGs, differentially expressed genes; CRC, colorectal cancer; q-value, adjusted P-value; $\mathrm{GO}$, Gene Ontology; BP, biological process; CC, cellular component; MF, molecular function.

addition, the high expression of TOP2A was revealed to be correlated with a worse survival in breast cancer, small-cell lung cancer and malignant melanoma, indicating that TOP2A can serve as a prognostic biomarker (39-41). TOP2A was also revealed to be associated with advanced CRC and chemotherapeutic resistance via the inhibition of apoptosis (35). Zhou et al (42) revealed that TOP2A involvement in T-cell factor transcription may transmit a mechanism of multidrug resistance to TOP2A inhibitors, which can be an effective treatment option for CRC. Therefore, further investigation is required to elucidate the mechanism of TOP2A in the development, progression and treatment of CRC. CDC6 is essential for the initiation of DNA replication and contains ATP-binding and hydrolytic activities, which are required for the formation of the pre-replicative complex $(43,44)$. It is also involved in the cell cycle by localizing to the centrosomes during the $\mathrm{S}$ and G2 phases (45). It has been reported that CDC6 affects proliferation during the early differentiation stages (46), and that the overexpression of CDC6 in tumors could signify that it may be an oncogenic target. CHEK1 is a serine/threonine kinase involved in delaying cell cycle progression; it is also required for the activation of DNA repair in response to the presence of DNA damage or unreplicated DNA (47). MAD2L1 (also termed MAD2) plays an important role in maintaining the spindle checkpoint function. MAD2L1 was revealed to be correlated with disease outcome in patients with breast cancer (48), and an increased MAD2L1 expression in neuroblastoma cells to be associated with poor prognosis (49).

The PPI network module analysis revealed that the development of CRC was mainly associated with the cell cycle 
Table V. KEGG pathway analysis of selected module-associated DEGs.

\begin{tabular}{lcll}
\hline KEGG & Count & q-value & \\
\hline Cell cycle & 6 & $1.88 \times 10^{-5}$ & BUB1B/CCNB1/CDC6/CHEK1/MAD2L1/TTK \\
Progesterone-mediated oocyte maturation & 4 & $7.64 \times 10^{-4}$ & AURKA/CCNB1/GNAI1/MAD2L1 \\
Chemokine signaling pathway & 9 & $4.69 \times 10^{-8}$ & CCL19/CCL28/CXCL1/CXCL12/CXCL3/CXCL5/ \\
& & & CXCL8/GNAI1/GNG4 \\
IL-17 signaling pathway & 4 & $9.02 \times 10^{-4}$ & CXCL1/CXCL3/CXCL5/CXCL8 \\
$\begin{array}{l}\text { Legionellosis } \\
\text { Rheumatoid arthritis }\end{array}$ & 3 & $9.37 \times 10^{-4}$ & CXCL1/CXCL3/CXCL8 \\
& 4 & $1.06 \times 10^{-3}$ & CXCL1/CXCL12/CXCL5/CXCL8
\end{tabular}

DEGs, differentially expressed genes; KEGG, Kyoto Encyclopedia of Genes and Genomes.

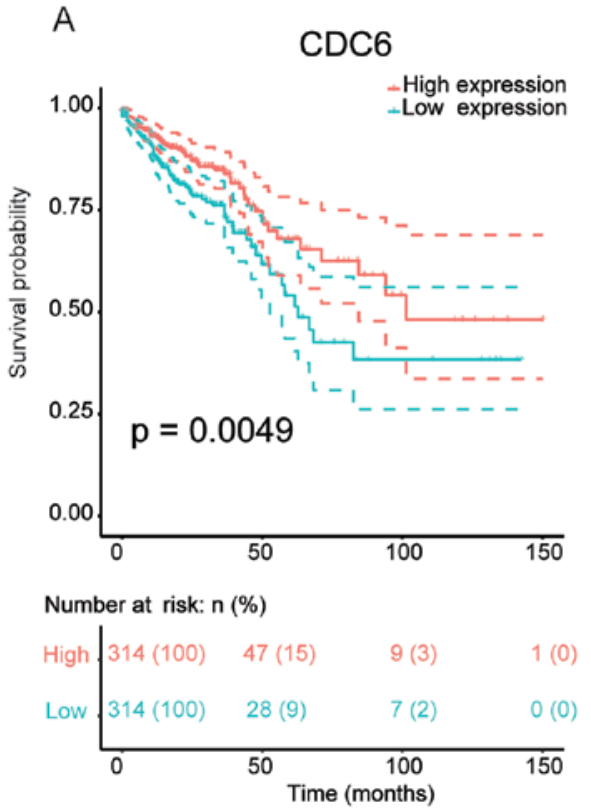

C

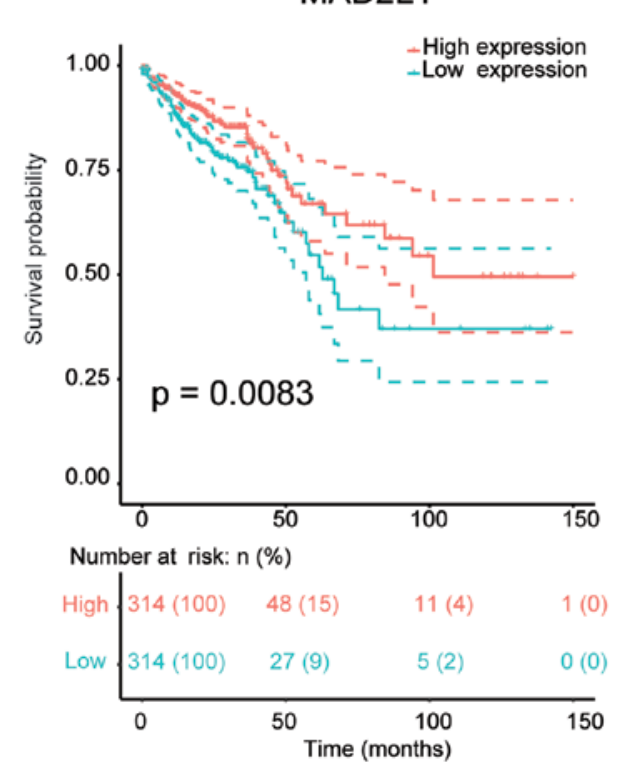

B
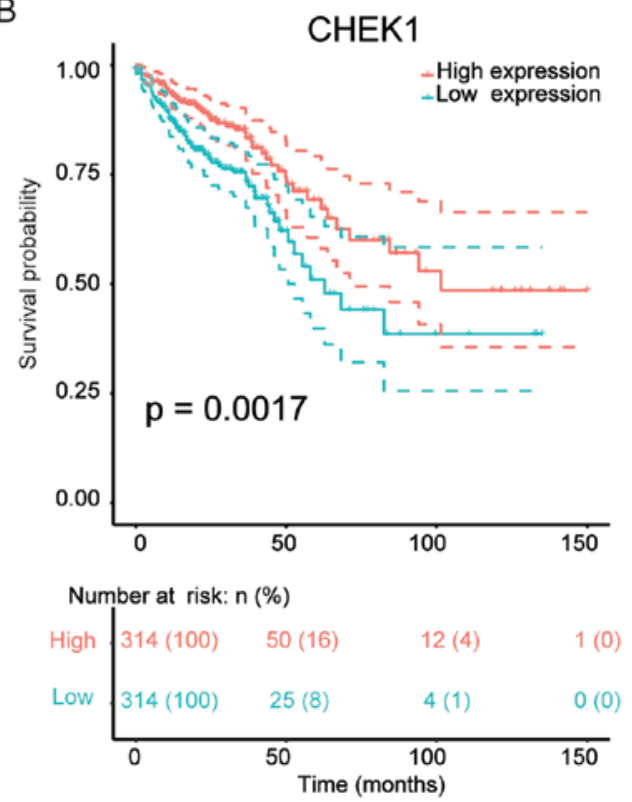

$\mathrm{D}$

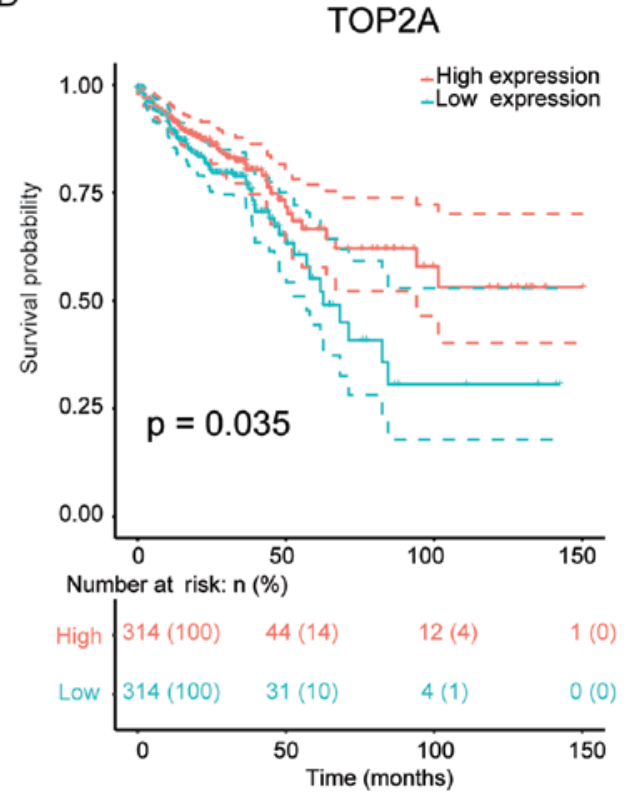

Figure 4. Survival analysis showing the correlation between the expression of hub genes and overall survival in CRC patients. (A) CDC6, (B) CHEK1, (C) MAD2L1 and (D) TOP2A. Red lines represent high and green lines low expression genes. Dashed lines represent CI. The X-axis shows the survival time of CRC patients and the y-axis the survival probability. CRC, colorectal cancer; CDC6, Cell Division Cycle 6; CHEK1, Checkpoint Kinase 1; MAD2L1, Mitotic Arrest Deficient 2 Like 1; TOP2A, Topoisomerase II Alpha; CI, confidence interval. 
pathway. It has been reported that cell cycle signaling pathways play a vital role in controlling normal progression, and regulating cell proliferation and apoptosis; an uncontrolled cell cycle may result in cancer (27). Therefore, cell cycle regulation is a useful way of interfering with the development of CRC.

Recent studies have indicated that mRNAs, miRNAs and IncRNAs play a crucial role in a variety of biological processes associated with human diseases $(50,51)$. Chen et al (52-55), have constructed powerful computational models to predict potential associations between miRNAs/lncRNAs and human diseases, providing a reliable and powerful tool for disease-association prediction. With the application of computational models, more stable and effective biomarkers (miRNAs/lncRNAs) would be revealed, and the pathogenesis of CRC would be explained at different molecular levels.

The present results provided useful information for early diagnosis and prevention, and supplied an effective therapeutic target for CRC. However, the study has certain limitations: i) By mixing all cancer types together, the focused insights in genetic characteristics of different subtypes of tumors may not be revealed, therefore, a stratification analysis of major clinical information should be performed; ii) more databases should be used for validation of the DEGs in future research; iii) in the present study, all tumor samples were included for the evaluation of the prognositic role of hub genes, however, it would be better to exclude CRC without matching normal controls, which would reduce the difference arising from different clinical information, and iv) intersect function analysis of GEO and TCGA was performed to screen DEGs, however, among these data sets, GSE22598 included colon cancer treated with chemotherapy which may affect the results, therefore, sample information should be more focused in future research. In addition, biological experiments are required to confirm these results. In conclusion, in the present study DEGs were identified by integrated bioinformatics analysis, in order to explore the role of DEGs in the progression and prognosis of CRC. Consequently, 405 DEGs were screened out, and hub genes CDC6, CHEK1, MAD2L1 and TOP2A were revealed to be promising prognostic biomarkers among CRC patients.

\section{Acknowledgements}

Not applicable.

\section{Funding}

The present study was supported by grants from the National Natural Science Foundation of China (grant nos. 81472922 and 81673105).

\section{Availability of data and materials}

All data generated or analyzed during the present study are included in this article, and the $\mathrm{R}$ codes are available at https://github.com/Yuchang66/CRC-project.

\section{Authors' contributions}

All authors conceived and designed the experiments. CY contributed to the bioinformatics analysis and drafted the manuscript. CY, FC and JJ contributed to the data mining. HZ and $\mathrm{MZ}$ contributed to the statistical analysis and modified the manuscript. All authors read and approved the final version of the manuscript and agree to be accountable for all aspects of the research in ensuring that the accuracy or integrity of any part of the work are appropriately investigated and resolved.

\section{Ethics approval and consent to participate}

Not applicable.

\section{Patient consent for publication}

Not applicable.

\section{Competing interests}

The authors declare that they have no competing interests.

\section{References}

1. Bray F, Ferlay J, Soerjomataram I, Siegel RL, Torre LA and Jemal A: Global cancer statistics 2018: GLOBOCAN estimates of incidence and mortality worldwide for 36 cancers in 185 countries. CA Cancer J Clin 68: 394-424, 2018.

2. O'Connell JB, Maggard MA and Ko CY: Colon cancer survival rates with the new American joint committee on cancer sixth edition staging. J Natl Cancer Inst 96: 1420-1425, 2004.

3. Aslinia F, Uradomo L, Steele A, Greenwald BD and Raufman JP: Quality assessment of colonoscopic cecal intubation: An analysis of 6 years of continuous practice at a university hospital. Am J Gastroenterol 101: 721-731, 2006.

4. Uraoka T, Ramberan H, Matsuda T, Fujii T and Yahagi N: Cold polypectomy techniques for diminutive polyps in the colorectum. Dig Endosc 26 (Suppl 2): S98-S103, 2014.

5. Martín-López JE, Beltrán-Calvo C, Rodríguez-López R and Molina-López T: Comparison of the accuracy of CT colonography and colonoscopy in the diagnosis of colorectal cancer. Colorectal Dis 16: O82-O89, 2014.

6. Liang B, Li C and Zhao J: Identification of key pathways and genes in colorectal cancer using bioinformatics analysis. Med Oncol 33: 111, 2016.

7. Guo Y, Bao Y, Ma M and Yang W: Identification of key candidate genes and pathways in colorectal cancer by integrated bioinformatical analysis. Int J Mol Sci 18: pii: E722, 2017.

8. Hong Y, Downey T, Eu KW, Koh PK and Cheah PY: A 'metastasis-prone' signature for early-stage mismatch-repair proficient sporadic colorectal cancer patients and its implications for possible therapeutics. Clin Exp Metastasis 27: 83-90, 2010.

9. Okazaki S, Ishikawa T, Iida S, Ishiguro M, Kobayashi $H$, Higuchi T, Enomoto M, Mogushi K, Mizushima H, Tanaka H, et al: Clinical significance of UNC5B expression in colorectal cancer. Int J Oncol 40: 209-216, 2012.

10. Barrett T, Wilhite SE, Ledoux P, Evangelista C, Kim IF, Tomashevsky M, Marshall KA, Phillippy KH, Sherman PM, Holko M, et al: NCBI GEO: Archive for functional genomics data sets-update. Nucleic Acids Res 41 (Database Issue): D991-D995, 2013.

11. Irizarry RA, Hobbs B, Collin F, Beazer-Barclay YD, Antonellis KJ, Scherf U and Speed TP: Exploration, normalization, and summaries of high density oligonucleotide array probe level data. Biostatistics 4: 249-264, 2003.

12. Ritchie ME, Phipson B, Wu D, Hu Y, Law CW, Shi W and Smyth GK: Limma powers differential expression analyses for RNA-sequencing and microarray studies. Nucleic Acids Res 43: e47, 2015.

13. Robinson MD, McCarthy DJ and Smyth GK: EdgeR: A bioconductor package for differential expression analysis of digital gene expression data. Bioinformatics 26: 139-140, 2010.

14. Oliveros JC: VENNY. An interactive tool for comparing lists with Venn Diagrams, 2007. 
15. Ashburner M, Ball CA, Blake JA, Botstein D, Butler $\mathrm{H}$, Cherry JM, Davis AP, Dolinski K, Dwight SS, Eppig JT, et al: Gene ontology: Tool for the unification of biology. The gene ontology consortium. Nat Genet 25: 25-29, 2000

16. The Gene Ontology Consortium: The gene ontology resource: 20 years and still GOing strong. Nucleic Acids Res 47 (D1): D330-D338, 2019.

17. Kanehisa M, Sato Y, Furumichi M, Morishima K and Tanabe M New approach for understanding genome variations in KEGG Nucleic Acids Res 47 (D1): D590-D595, 2019.

18. Kanehisa M and Goto S: KEGG: Kyoto encyclopedia of genes and genomes. Nucleic Acids Res 28: 27-30, 2000.

19. Kanehisa M, Furumichi M, Tanabe M, Sato Y and Morishima K: KEGG: New perspectives on genomes, pathways, diseases and drugs. Nucleic Acids Res 45 (D1): D353-D361, 2017.

20. Yu G, Wang LG, Han Y and He QY: clusterProfiler: An R package for comparing biological themes among gene clusters. OMICS 16: 284-287, 2012

21. Yu G, Wang LG, Yan GR and He QY: DOSE: An R/Bioconductor package for disease ontology semantic and enrichment analysis. Bioinformatics 31: 608-609, 2015

22. Shannon P, Markiel A, Ozier O, Baliga NS, Wang JT, Ramage D, Amin N, Schwikowski B and Ideker T: Cytoscape: A software environment for integrated models of biomolecular interaction networks. Genome Res 13: 2498-2504, 2003.

23. Bader GD and Hogue CW: An automated method for finding molecular complexes in large protein interaction networks. BMC Bioinformatics 4: 2, 2003

24. Bindea G, Mlecnik B, Hackl H, Charoentong P, Tosolini M Kirilovsky A, Fridman WH, Pagès F, Trajanoski Z and Galon J: ClueGO: A cytoscape plug-in to decipher functionally grouped gene ontology and pathway annotation networks. Bioinformatics 25: 1091-1093, 2009.

25. Chin $\mathrm{CH}$, Chen $\mathrm{SH}, \mathrm{Wu} \mathrm{HH}, \mathrm{Ho} \mathrm{CW}$, Ko MT and Lin CY: CytoHubba: Identifying hub objects and sub-networks from complex interactome. BMC Syst Biol 8 (Suppl 4): S11, 2014.

26. Takada I and Makishima M: Control of inflammatory bowel disease and colorectal cancer by synthetic vitamin D receptor ligands. Curr Med Chem 24: 868-875, 2017.

27. Vermeulen K, Van Bockstaele DR and Berneman ZN: The cell cycle: A review of regulation, deregulation and therapeutic targets in cancer. Cell Prolif 36: 131-149, 2010.

28. Aarts M, Linardopoulos S and Turner NC: Tumour selective targeting of cell cycle kinases for cancer treatment. Curr Opin Pharmacol 13: 529-535, 2013.

29. Rieder G, Hofmann JA, Hatz RA, Stolte M and Enders GA: Up-regulation of inducible nitric oxide synthase in helicobacter pylori-associated gastritis may represent an increased risk factor to develop gastric carcinoma of the intestinal type. Int J Med Microbiol 293: 403-412, 2003

30. Hashibe M, Galeone C, Buys SS, Gren L, Boffetta P, Zhang ZF and La Vecchia C: Coffee, tea, caffeine intake, and the risk of cancer in the PLCO cohort. Br J Cancer 113: 809-816, 2015.

31. Guertin KA, Loftfield E, Boca SM, Sampson JN, Moore SC, Xiao Q, Huang WY, Xiong X, Freedman ND, Cross AJ and Sinha R: Serum biomarkers of habitual coffee consumption may provide insight into the mechanism underlying the association between coffee consumption and colorectal cancer. Am J Clin Nutr 101: 1000-1011, 2015.

32. Merighi S, Benini A, Mirandola P, Gessi S, Varani K, Simioni C, Leung E, Maclennan S, Baraldi PG and Borea PA: Caffeine inhibits adenosine-induced accumulation of hypoxia-inducible factor-1alpha, vascular endothelial growth factor, and interleukin-8 expression in hypoxic human colon cancer cells. Mol Pharmacol 72: 395-406, 2007.

33. Long Y, Sanchez-Espiridion B, Lin M, White L, Mishra L, Raju GS, Kopetz S, Eng C, Hildebrandt MAT, Chang DW, et al: Global and targeted serum metabolic profiling of colorectal cancer progression. Cancer 123: 4066-4074, 2017.

34. Chen L, Lu D, Sun K, Xu Y, Hu P, Li X and Xu F: Identification of biomarkers associated with diagnosis and prognosis of colorectal cancer patients based on integrated bioinformatics analysis. Gene 692: 119-125, 2019.

35. Coss A, Tosetto M, Fox EJ, Sapetto-Rebow B, Gorman S, Kennedy BN, Lloyd AT, Hyland JM, O'Donoghue DP, Sheahan K, et al: Increased topoisomerase IIalpha expression in colorectal cancer is associated with advanced disease and chemotherapeutic resistance via inhibition of apoptosis. Cancer Lett 276: 228-238, 2009.
36. Bofin AM, Ytterhus B and Hagmar BM: TOP2A and HER-2 gene amplification in fine needle aspirates from breast carcinomas. Cytopathology 14: 314-319, 2003

37. Kanta SY, Yamane T, Dobashi Y, Mitsui F, Kono K and Ooi A: Topoisomerase IIalpha gene amplification in gastric carcinomas: Correlation with the HER2 gene. An immunohistochemical, immunoblotting, and multicolor fluorescence in situ hybridization study. Hum Pathol 37: 1333-1343, 2006.

38. Kruger S, Lange I, Kausch I and Feller AC: Protein expression and gene copy number analysis of topoisomerase 2alpha, HER2 and P53 in minimally invasive urothelial carcinoma of the urinary bladder-a multitissue array study with prognostic implications. Anticancer Res 25: 263-271, 2005.

39. Depowski PL, Rosenthal SI, Brien TP, Stylos S, Johnson RL and Ross JS: Topoisomerase IIalpha expression in breast cancer: Correlation with outcome variables. Mod Pathol 13: 542-547, 2000.

40. Dingemans AM, Witlox MA, Stallaert RA, van der Valk P, Postmus PE and Giaccone G: Expression of DNA topoisomerase IIalpha and topoisomerase IIbeta genes predicts survival and response to chemotherapy in patients with small cell lung cancer. Clin Cancer Res 5: 2048-2058, 1999.

41. Mu XC, Tran TA, Ross JS and Carlson JA: Topoisomerase II-alpha expression in melanocytic nevi and malignant melanoma. J Cutan Pathol 27: 242-248, 2000.

42. Zhou Q, Abraham AD, Li L, Babalmorad A, Bagby S, Arcaroli JJ, Hansen RJ, Valeriote FA, Gustafson DL, Schaack J, et al: Topoisomerase II $\alpha$ mediates TCF-dependent epithelial-mesenchymal transition in colon cancer. Oncogene 35: 4990-4999, 2016.

43. Fernandez-Cid A, Riera A, Tognetti S, Herrera MC, Samel S, Evrin C, Winkler C, Gardenal E, Uhle S, Speck C, et al: An $\mathrm{ORC} / \mathrm{Cdc} 6 / \mathrm{MCM} 2-7$ complex is formed in a multistep reaction to serve as a platform for MCM double-hexamer assembly. Mol Cell 50: 577-588, 2013.

44. Randell JC, Bowers JL, Rodriguez HK and Bell SP: Sequentia ATP hydrolysis by Cdc6 and ORC directs loading of the Mcm 2-7 helicase. Mol Cell 21: 29-39, 2006.

45. Kim GS, Kang J, Bang SW and Hwang DS: Cdc6 localizes to $\mathrm{S}$ - and G2-phase centrosomes in a cell cycle-dependent manner. Biochem Biophys Res Commun 456: 763-767, 2015.

46. Barkley LR, Hong HK, Kingsbury SR, James M, Stoeber K and Williams GH: Cdc6 is a rate-limiting factor for proliferative capacity during HL60 cell differentiation. Exp Cell Res 313: 3789-3799, 2007

47. Huang M, Miao ZH, Zhu H, Cai YJ, Lu W and Ding J: Chk1 and Chk2 are differentially involved in homologous recombination repair and cell cycle arrest in response to DNA double-strand breaks induced by camptothecins. Mol Cancer Ther 7: 1440-1449, 2008.

48. van't Veer LJ, Dai H, van de Vijver MJ, He YD, Hart AA, Mao M, Peterse HL, van der Kooy K, Marton MJ, Witteveen AT, et al: Gene expression profiling predicts clinical outcome of breast cancer. Nature 415: 530-536, 2002.

49. Hernando E, Nahle Z, Juan G, Diaz-Rodriguez E, Alaminos M, Hemann M, Michel L, Mittal V, Gerald W, Benezra R, et al: Rb inactivation promotes genomic instability by uncoupling cell cycle progression from mitotic control. Nature 430: 797-802, 2004.

50. Chen X, Yan CC, Zhang X and You ZH: Long non-coding RNAs and complex diseases: From experimental results to computational models. Brief Bioinform 18: 558-576, 2017.

51. Chen X, Xie D, Zhao Q and You ZH: MicroRNAs and complex diseases: From experimental results to computational models. Brief Bioinform 20: 515-539, 2019.

52. Chen X, Wang L, Qu J, Guan NN and Li JQ: Predicting miRNA-disease association based on inductive matrix completion. Bioinformatics 34: 4256-4265, 2018.

53. Chen X, Xie D, Wang L, Zhao Q, You ZH and Liu H: BNPMDA: Bipartite network projection for MiRNA-disease association prediction. Bioinformatics 34: 3178-3186, 2018.

54. Chen X, Yin J, Qu J and Huang L: MDHGI: Matrix decomposition and heterogeneous graph inference for miRNA-disease association prediction. PLoS Computat Biol 14: e1006418, 2018.

55. Chen $X$ and Huang L: LRSSLMDA: Laplacian regularized sparse subspace learning for MiRNA-disease association prediction. PLoS Comput Biol 13: e1005912, 2017.

This work is licensed under a Creative Commons Attribution-NonCommercial-NoDerivatives 4.0 International (CC BY-NC-ND 4.0) License. 